\title{
Weight gain in Association with Insulin Use - An Analysis of Individual Case Safety Reports an Indian Database
}

Prasad Thota ${ }^{1 *}$, Kalaiselvan Vivekanandan ${ }^{1}$, Jai Prakash ${ }^{1}$, Surinder Singh ${ }^{2}$ and Gyanendra Nath Singh ${ }^{1}$

${ }^{1}$ National Coordination Centre, Pharmacovigilance Programme of India, Indian Pharmacopoeia Commission, Ghaziabad, Uttar Pradesh, India

${ }^{2}$ National Institute of Biologicals, Noida, India

\begin{abstract}
Background: The Central Drugs Standard Control Organization's (CDSCO) Pharmacovigilance Programme of India for National Drug Monitoring, maintained by the National Coordination Centre (NCC)- Indian Pharmacopoeia Commission, has 60 member AMCs (Adverse drug reaction Monitoring Centers) contributing individual case safety reports (ICSRs) from their existing regional Pharmacovigilance centers; these reports are stored in the Indian patients ICSR database, VigiFlow. A continuous increase of ICSRs with weight-increase is suspected to be connected with insulin use has been observed in VigiFlow; however only limited information has been published on this topic.
\end{abstract}

Objective: To examine in detail the association between Insulin and weight gain by outlining the characteristics of the accumulated reports in VigiFlow.

Method: An analysis of 24,006 ICSRs in VigiFlow, from 15 April 2011 to 31 October 2012, where insulin was suspected of causing weight gain.

Results: Insulin has been suspected of being involved in the development of weight gain in 33 patients, as reported by 60 AMCs. The age of the patients ranged between 15 years to 73 years (mean 37 years), with a predominance $(56 \%)$ of patients $>30$ years of age and 32 patients were male. In 33 reports, Insulin was the only drug suspected by the reporter, and in all 33 reports, Insulin was the sole reported drug. Commonly concomitant drugs were other oral anti diabetic drugs. In 33 patients, weight gain has reported as the single event and the relation between the drug and reaction was possible according to WHO causality scale. None of the patients were reported to have had a recurrence of weight gain on re-administration of Insulin.

Conclusions: The NCC continues to receive reports of weight gain associated with the use of Insulin. Although weight gain may not be regarded as serious from a regulatory perspective, this adverse reaction has the potential to affect compliance resulting in decreased efficacy of the treatment regimen and detrimental effects on patient health outcomes.

Keywords: Weight gain; Insulin; Indian database; Individual case safety reports

\section{Introduction}

A continuous increase of ICSRs with weight gain in suspected to be connected with insulin use has been observed in the Indian patients ICSRs database i.e. VigiFlow. However, nine case reviews performed by the USA diabetes care in 1994 [1], Diabetes Control and Complications Trial in 1998 [2], UK Prospective Diabetes Study (UKPDS) group in 1998 [3], UK diabetes care in 1999 [4], Hermansen and Mortensen [5], and four reviews [6-9] are publicly available on this topic. The CDSCO Programme for Pharmacovigilance maintained by National Coordination Centre-Indian Pharmacopeia Commission, have 60 adverse drug reactions Monitoring Centres (AMCs). The present work triggered a review of all reports received nationally by the Pharmacovigilance Programme of India. The centers contribute ICSRs from their existing regional pharmacovigilance systems and these reports are stored in VigiFlow. The reports contain divergent data with respect to center of report origin, reporter type, amount of data and quality of data. The reports represent a varying degree of suspicion and as with all spontaneous reporting data the results must be interpreted with caution. One of the main tasks of the NCC-PvPI is to issue new signals or unknown ADR for drug safety concerns arising from the data and case summaries are regularly distributed to the member adverse reaction monitoring centres (AMCs) in India and World Health Organization- Uppsala Monitoring Centre (WHO-UMC) [10].

Insulin, a polypeptide hormone, is an antidiabetic medicine used as monotherapy or as an adjunct to treatment with other oral antidiabetic drugs, e.g. metformin, pioglitazone [11-13]. It is used as first-line treatment for Insulin-Dependent Diabetes Mellitus (IDDM) and also used as intermittent or permanent therapy in Non Insulin-Dependent Diabetes Mellitus (NIDDM) [14]. Hypoglycemia, lipodystrophy, sweating, dizziness, hunger, pallor, headache, visual disturbances, injection site reactions are known adverse drug reactions (ADRs) for Insulin [11]. This is an initial evaluation of cases with insulin and weight gain undertaken by NCC in 2012. The review indicated that weight gain could be another ADR, although less serious than hypoglycemia associated with insulin use.

The assessment based on 33 reports, was distributed among the members of the National Pharmacovigilance programme, via the restricted document SIGNAL (unpublished data) [10]. In December 2012, when a follow-up on the topic was performed, weight gain was listed as maybe an ADR for insulin in Martindale [11], India and UK

*Corresponding author: Prasad Thota, National Coordination Center Pharmacovigilance Programme of India, Indian Pharmacopoeia Commission, Ghaziabad, Uttar Pradesh, India, E-mail: prasad.thota187@gmail.com

Received July 02, 2013; Accepted January 30, 2014; Published February 07 2014

Citation: Thota P, Vivekanandan K, Prakash J, Singh S, Singh GN (2014) Weight gain in Association with Insulin Use - An Analysis of Individual Case Safety Reports an Indian Database. J Pharmacovigilance 2: 119. doi:10.4172/2329-6887.1000119

Copyright: $\odot 2014$ Thota P, et al., This is an open-access article distributed under the terms of the Creative Commons Attribution License, which permits unrestricted use, distribution, and reproduction in any medium, provided the original author and source are credited. 


\begin{tabular}{|c|c|}
\hline Age range $(\mathbf{y})$ & No. of patients \\
\hline$<20$ & 04 \\
\hline $21-30$ & 11 \\
\hline $31-40$ & 05 \\
\hline $41-50$ & 07 \\
\hline $51-60$ & 04 \\
\hline$>60$ & 02 \\
\hline
\end{tabular}

Table 1: Patient age for Insulin and weight gain reports.

\begin{tabular}{|c|c|}
\hline Type of Diabetes & No. of Reports \\
\hline Type-1 & 13 \\
\hline Type-2 & 09 \\
\hline Unspecified & 11 \\
\hline
\end{tabular}

Table 2: Type of Diabetes for Insulin and weight gain reports

\begin{tabular}{|c|c|}
\hline Reported Term & No. of Report \\
\hline Weight Increase & 30 \\
\hline Obesity & 03 \\
\hline a Reported terms are listed as originally coded on the report either by WHO \\
Adverse Reaction Terminology included terms or by Medical Dictionary for \\
Regulatory Activities lowest level terms. \\
b one report can contain more than one term.
\end{tabular}

Table 3: Reported weight gain terms.

\begin{tabular}{|c|c|}
\hline Co-reported reaction & No. of Reports \\
\hline Hypoglycaemia & 193 \\
\hline Lipodystrophy & 39 \\
\hline Giddiness & 15 \\
\hline Sweating increased & 05 \\
\hline Hypokalemia & 03 \\
\hline Obesity & 03 \\
\hline Vertigo & 02 \\
\hline Loss of appetite & 02 \\
\hline Vision abnormal & 01 \\
\hline Pruritus & 01 \\
\hline
\end{tabular}

Table 4: The ten most frequently co-reported adverse reactions by WHO Adverse Reaction Terminology preferred term for Insulin and weight gain reports.

Summary of Product Characteristics (SPC) [12] or Drugdex [11]. In the Physician's Desk Reference (PDR) [15] weight gain was stated to have been infrequently reported as an adverse event in clinical trials.

The aim of this study is to communicate more details on association between insulin and weight gain to a broader audience by outlining the characteristics of accumulated reports in VigiBase. Although weight gain from a regulatory perspective is not an $\mathrm{ADR}$ of serious character, it may have negative consequences on the compliance of the antidiabetic medicine use and thereby cause patient harm.

\section{Method}

All 24,006 ICSRs in VigiFlow from 15 April 2011 to 31 October 2012 were reviewed and descriptive data was analyzed retrospectively where insulin was suspected to cause weight increase (WHO Adverse Reaction Terminology [WHO-ART] preferred term).

\section{Results}

As of $31^{\text {st }}$ October, 2012 the number of ICSRs in VigiFlow in which insulin has been suspected of being involved in the development of weight gain in 33 patients, as reported by 60 AMCs since April 2011. Weight gain was reported disproportionately more often for insulin compared with the overall reporting of weight gain in VigiFlow, with an Proportionality Reporting Ratio (PRR) value of 5.08 [16] lower and upper limit of the 95\% confidence interval for the PRR were 1.294 and 1.959 , respectively. At the time of the study, VigiFlow contained a total of 316 reports of Insulin induced ADRs. Patient's demographics showed that $32(97 \%)$ patients were male and average age was 37 years, with a range between 15 to 73 years. The distribution is presented in Table 1 . Although 60 AMCs has reported weight gain in suspected connection with Insulin use, maximum reports reported from south regional pharmacovigilance AMCs of India. Distribution of Diabetes cases for insulin associated weight gain as seen in Table 2.

\section{Reported term}

WHO-ART terminology groups similar terms and each report may contain a more detailed term. Specific of the reported lower level weight increase terms are provided in Table 3. One report can include more than one term.

\section{Co-reported drugs}

In 33 reports insulin was the only drug suspected by the reporter and 225 reports included co-reported concomitant or suspected drugs (each report can contain several co reported drugs). The most frequently co-reported drugs (as either suspected, interacting or concomitant) each appearing in more than ten reports are displayed in Table 4. Five of the top co-reported drugs are antidiabetic drugs commonly used together with insulin.

\section{Co-reported events}

For 33 patients, weight gain was reported as the single event. The 10 most frequently co-reported adverse reactions are displayed in Table 5. Apart from weight gain, a few reports also included weight gain-related terms, such as obesity $(n=3)$. The majority of the co-reported metabolic and nutritional reactions (267 events in 277 reports) were not serious, such as hypoglycemia (153 events, 154 reports), the only co-reported adverse reactions of a more serious nature (those on the WHO-ART critical terms list) are listed in Table 6.

\section{Time to onset}

Details on time from Insulin start to onset weight gain were available and categorized in 33 cases.

\section{Reports with Information on dechallenge and rechallenge}

For all 33 patients the drug was withdrawn.

\begin{tabular}{|c|c|}
\hline Co-reported serious metabolic reaction & No. of Reports \\
\hline Hypoglycemia & 40 \\
\hline Increased sweating & 4 \\
\hline Giddiness & 2 \\
\hline Appetite loss & 2 \\
\hline Burning sensation & 1 \\
\hline
\end{tabular}

Table 5: Co-reported serious skin reactions according to WHO Adverse Reaction Terminology critical preferred terms for Insulin and weight gain reports.

\begin{tabular}{|c|c|}
\hline Time to onset category & $\begin{array}{c}\text { No. of reports where Insulin was the } \\
\text { sole-suspected drug }\end{array}$ \\
\hline$<1 \mathrm{yr}$ & 11 \\
\hline $1-2 \mathrm{yrs}$ & 11 \\
\hline $2-3 \mathrm{yrs}$ & 05 \\
\hline $3-4$ yrs & 03 \\
\hline$>4$ yrs & 02 \\
\hline Unknown & 01 \\
\hline
\end{tabular}

Table 6: Reported time to onset for Insulin and weight gain reports. 
Citation: Thota P, Vivekanandan K, Prakash J, Singh S, Singh GN (2014) Weight gain in Association with Insulin Use - An Analysis of Individual Case Safety Reports an Indian Database. J Pharmacovigilance 2: 119. doi:10.4172/2329-6887.1000119

Page 3 of 3

\section{Adverse reaction outcome information}

Outcome information was available for 22 patients where outcome of reaction was unknown and for rest of 11 reports were unspecified in report.

\section{Discussion}

Since 2011 weight gain has been reported in suspected connection with insulin use from 60 centres under the Pharmacovigilance Programme of India for monitoring drug safety. In the majority of these cases reporter had listed Insulin to be the single suspected drug. Maximum of ICSRs were reported by health care professionals, which implies that weight gain is a concern felt strongly enough by physician that they report ADR, but which consumers or non healthcare professionals may over look or be insufficiently motivated to report. Reports were also received from doctors, pharmacists and other health professionals indicating that these events were also regarded as significant by healthcare professionals. The review of patient demographics shows that weight gain is reported for patients of all ages, but with a predominance $(56 \%)$ of patients $>30$ years of age. A majority of the reports concerned males (97\%). Drug-induced weight gain seems to occur more often in men than women, and the patients generally do not have other co-occurring symptoms. Weight gain was reported as the only term in all of the cases and none of the patient was not reported weight gain as a serious event in addition to Insulin. Drug-induced weight gain is usually irreversible after discontinuation of treatment. The incidence and severity depend on the drug and individual predisposition. In general a causal association between a drug and a suspected adverse reaction is strengthened if there is improvement after withdrawing the drug (dechallenge) [17]. The causal association is further strengthened weight gain were available and categorized in 33 cases (see Table 6). Doses of insulin dosage information were available in 31 reports. For 31 patients a dosage above 20 IU/day was recorded of which three reports included a dosage above $50 \mathrm{IU} /$ day (the usual maintenance daily dose).

\section{Study Limitations}

The main limitation of this study is inherent to the study design. The results are only useful for implementing future pharmacovigilance actions. The present results are only a statistical sample size of the reports of only 2 years (2011 and 2012) and it is not intended that any generalizations beyond India are made.

\section{Conclusions}

The continuing increase in spontaneous reports and published case reports may provide sufficient support to include weight gain consistently in the product labeling for insulin in all countries where it is marketed. We believe it is important to increase awareness among physicians and patients since this adverse reaction has the potential to affect compliance, resulting in decreased efficacy of the treatment regimen and detrimental effects on patient safety.

\section{Acknowledgements}

The authors are indebted to the national coordination centres contributing data to the WHO Programme for International Drug Monitoring. The opinions and conclusions, however, are not necessarily those of either the various centres or the WHO. No sources of funding were used to assist in the preparation of this study. The authors have no conflicts of interest that are directly relevant to the content of this study.

\section{References}

1. Polonsky WH, Anderson BJ, Lohrer PA, Aponte JE, Jacobson AM, et al. (1994) Insulin omission in women with IDDM. Diabetes Care 17: 1178-1185.

2. Purnell JQ, Hokanson JE, Marcovina SM, Steffes MW, Cleary PA, et al. (1998) Effect of excessive weight gain with intensive therapy of type 1 diabetes on lipid levels and blood pressure: results from the DCCT. Diabetes Control and Complications Trial. JAMA 280: 140-146.

3. (1998) Intensive blood-glucose control with sulphonylureas or insulin compared with conventional treatment and risk of complications in patients with type 2 diabetes (UKPDS 33). UK Prospective Diabetes Study (UKPDS) Group. Lancet 352: 837-853.

4. Bryden KS, Neil A, Mayou RA, Peveler RC, Fairburn CG, et al. (1999) Eating habits, body weight, and insulin misuse. A longitudinal study of teenagers and young adults with type 1 diabetes. Diabetes Care 22: 1956-1960.

5. Hermansen K, Mortensen LS (2007) Bodyweight changes associated with antihyperglycaemic agents in type 2 diabetes mellitus. Drug Saf 30: 1127-1142.

6. Colditz GA, Willett WC, Rotnitzky A, Manson JE (1995) Weight gain as a risk factor for clinical diabetes mellitus in women. Ann Intern Med 122: 481-486.

7. Brancati FL, Wang NY, Mead LA, Liang KY, Klag MJ (1999) Body weight patterns from 20 to 49 years of age and subsequent risk for diabetes mellitus: the Johns Hopkins Precursors Study. Arch Intern Med 159: 957-963.

8. Wannamethee SG, Shaper AG (1999) Weight change and duration of overweight and obesity in the incidence of type 2 diabetes. Diabetes Care 22 : 1266-1272.

9. Anderson JW, Kendall CW, Jenkins DJ (2003) Importance of weight management in type 2 diabetes: review with meta-analysis of clinical studies. $J$ Am Coll Nutr 22: 331-339.

10. Vigiflow (2012) The Indian patients ICSR database system PharmacovigilanceProgramme of India News letter 2: 3-4.

11. Martindale. Thomson Micromedex database [online].

12. Summary of product characteristics for Insulin. Electronic medicine compendium, Datapharm Communications Ltd.

13. Fauci A, Braunwald E, Kasper D, Hauser S, Longo D, et al. (2008) (17 thedn) Harrison's Principles of Internal Medicine. The McGraw-Hill Companies Inc. New York.

14. http://www.bio.davidson.edu/courses/immunology/Students/spring2000/weed/ restricted/paper.html

15. Physician's desk reference, Thomson Micromedex database. Insulin S.C Injection [online].

16. European Medicines Agency: Evaluation of Medicines for Human Use (2006) Guideline On The Use Of Statistical Signal Detection Methods In The Eudravigilance Data Analysis System, London, UK.

17. Russell-Jones D, Khan R (2007) Insulin-associated weight gain in diabetes-causes, effects and coping strategies. Diabetes Obes Metab 9: 799-812. 